\title{
Articulation in children with fetal alcohol syndrome
}

\author{
Christopher L. Bolinger', James Dembowski \\ ${ }^{1}$ Texas Woman's University, Communication Sciences and Disorders, Denton, TX; ${ }^{2} T e x a s$ Tech University Health Sciences Center, Department of \\ Speech Language Hearing Sciences, Lubbock, TX, USA
}

Purpose: Language deficits have been identified in children with Fetal Alcohol Syndrome (FAS); however, literature on speech development is limited. Previous literature has documented neural abnormalities that impact motor control. Children diagnosed with FAS have slower response time, slower movement duration time, and greater variability relative to neuro-typical children. The purpose of the current study was to investigate presence/absence of motor speech dysfunction in children with FAS.

Methods: Ten children ages 3-9 years old were evaluated using standardized assessments for non-verbal intelligence, articulation, and verbal motor skills.

Results: Average group scores on a standardized articulation test (SPAT-D) did not produce significant differences; however, motor control subtests of the Verbal Motor Production Assessment for Children suggested significant differences between groups and matched participant pairs.

Conclusions: Results suggest specific oral motor deficits contribute at least as much to communication disorder in young FAS speakers as possible phonetic-linguistic deficits.

Keywords: Alcohol, Speech, Language, FASD, FAS, FAE

\section{INTRODUCTION}

Descriptions of the effects of prenatal alcohol exposure first appeared in the medical literature in the late 1960s and early 1970s [1-3]. Since then, these effects have become known as fetal alcohol syndrome (FAS). FAS is a medical diagnosis that refers to the physical malformations and cognitive impairments that occur from prenatal exposure to alcohol. Abnormalities may include prenatal growth deficiencies, postnatal growth deficiencies, developmental delays, craniofacial anomalies, limb malformations, finemotor malfunctions, external genitalia malformations, and pulmonary anomalies. Central nervous system dysfunctions include cognitive impairment and impaired motor function relative to developmental age [4]. According to Dorrie et al. [5], prenatal alcohol exposure is the greatest preventable risk contributing to neurologic abnormalities in children. Studies estimate the prevalence of fetal alcohol syndrome and additional alcohol related birth defects to be 24 to 48 per 1,000 U.S. live births [6]. This is approximately $2.4-4.8 \%$ of new births per year. According to National Vital Statistics Reports [7], there were 3,978,497 births. This means that there were a probable 95,484190,968 children born with fetal alcohol syndrome or alcohol related birth defects in that year, based on estimated prevalence rates. Incidence of FAS in the United States varies from 0.26 cases per 1,000 births in Caucasian Americans to 9.80 cases per 1,000

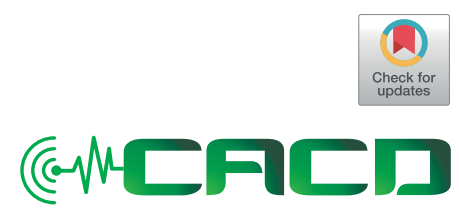

Received: January 15, 2019

Revision: March 23, 2019

Accepted: March 26, 2019

Correspondence:

Christopher L. Bolinger

Texas Woman's University, Communication Sciences and Disorders, P.O. Box 425737, Denton, TX 762045737, USA

Tel: $+940-898-2001$

Fax: +940-898-2070

E-mail: cbolinger@twu.edu

C 2019 The Korean Association of SpeechLanguage Pathologists

This is an Open Access article distributed under the terms of the Creative Commons Attribution NonCommercial License (http://creativecommons.org/ licenses/by-nc/4.0/) which permits unrestricted noncommercial use, distribution, and reproduction in any medium, provided the original work is properly cited. 
births in isolated Native American tribes [6].

With respect to communication, a growing literature has identified language deficits in children with FAS, particularly as language complexity increases [8-10]. In a recently published meta-analysis, Popova et al. [11], found a pooled prevalence rate of $67.2 \%$ for an unspecified developmental disturbance of speech and language (ICD-10 F80.9). The literature specific to speech development is more limited. Becker et al. [12] studied eight Native American Indian children under the age of 10. Three of the eight children with FAS exhibited articulatory abnormalities that were inconsistent with their mental age. The authors suggested a correlation between prenatal alcohol exposure and speech/language deficits in the areas of oral motor, articulation, short-term memory, grammatic and semantic abilities. Church et al. [13] studied 22 participants with a medical diagnosis of FAS in the age range of 3:8 to 26:11 years old. Eighteen participants presented with speech disorders including articulation errors, poor intelligibility, dysfluencies, hypernasality, and poor word formulation. Bolinger \& Dembowski's [14] study supported the theory of specific speech motor control deficits being a comorbidity within this population. Greater increases, variability, and differences in acoustic data indicated specific speech motor control deficits in FAS children relative to matched neurotypical children. The present study sought to further examine specific speech and oral motor function in children with FAS, with an emphasis on motor difficulties that may affect a child's articulation. Disambiguating the linguistic (phonologic) aspect from the motoric aspect of speech articulation remains a challenge in speech-language [15].

\section{METHODS}

\section{Participants}

Experimental participants were five children between three and ten years of age with a medical diagnosis of FAS, who were referred through the West Texas FASD Diagnostic Team. Experimental subjects were matched with five normal controls on mental age, socioeconomic status and gender.

\section{Testing procedures}

Participants were administered three standardized tests, in addition to a hearing screening and a child-friendly oral-motor screening. The three standardized tests were the Primary Test of Nonverbal Intelligence (PTONI), the Structured Photographic Articulation Test featuring Dudsberry (SPAT-D), and the Verbal Motor Production Assessment for Children (VMPAC). The PTONI was used to determine mental age. The SPAT-D is an articulation test which elicits 59 consonant phonemes and 10 consonant clusters at the word level. The VMPAC assesses speech tasks and related oral-motor function in five areas: general motor control, focal oromotor control, sequencing, connected speech and language control, and speech characteristics. Scores from the two speech tests were converted to percentile ranks and standard scores (mean= 100) for comparison across tests and participants. The independent variable in the study was subject classification as typically developing or diagnosed with FAS. Dependent variables were scores on the two standardized speech tests. Group differences were examined statistically with non-parametric Wilcoxon Ranksum tests.

\section{RESULTS}

Average chronological age for the experimental group was 7.95 years; average age for the control group was 5.96 years. Chronological ages of the two groups differed significantly (ranksum $=36, p=0.0476$ ). Based on PTONI scores, mental ages for the two groups did not differ significantly. Average mental age for the experimental group was 5.77 years, and 6.31 years for the control group $(p>0.05)$. Figure 1 shows the differences between chronological age and mental age for the two groups. There was no essential difference between chronological and mental age for the control group, and difference values among individuals varied minimally. Mental age for the experimental group was a little more than two years below chronological age, and difference scores among individuals

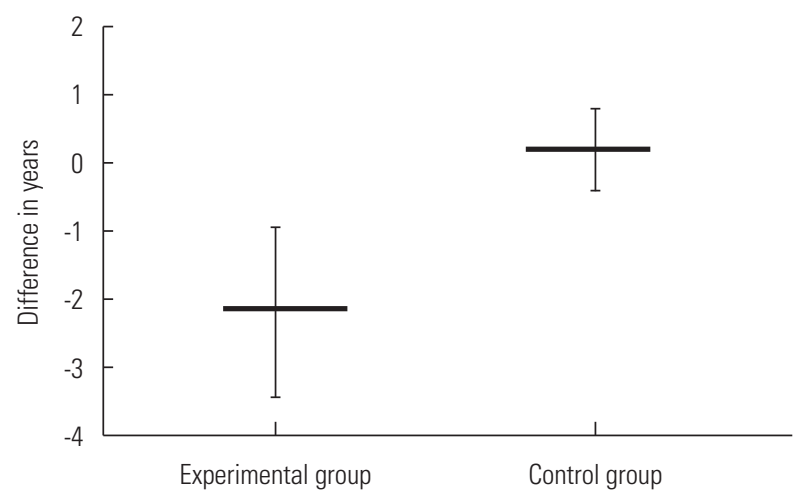

Figure 1. Mean difference between chronological age and mental age for each group. Chronological and mental age were substantially the same for control participants. Mental age for FAS speakers was just over 2 years below chronological age. FAS, fetal alcohol syndrome. 


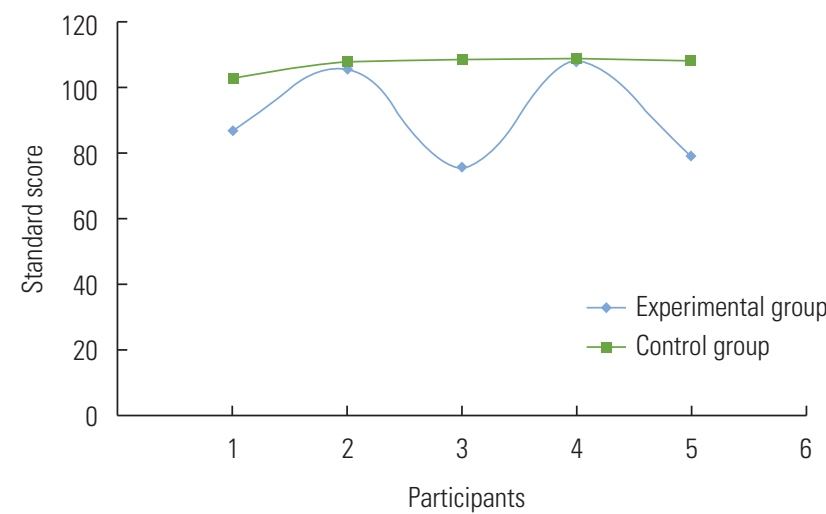

Figure 2. SPAT-D standard scores for each mental-aged matched participant pair. SPAT-D, structured photographic articulation test - featuring dudsberry.

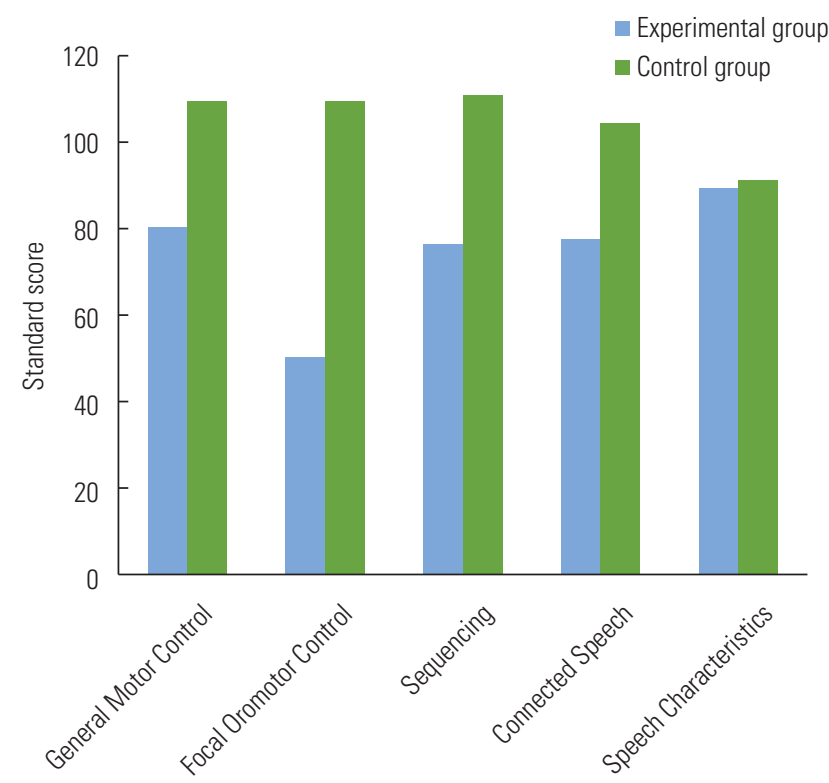

Figure 3. Mean group standard scores on each subtest of the VMPAC. The experimental group performed below average (100) on every subtest. Significant group differences were found for General Motor, Focal Oromotor, and Sequencing subtests. VMPAC, verbal motor production assessment for children.

varied more than for the control group.

Figure 2 shows SPAT-D articulation scores for each mentalage matched pair of participants, expressed as standard scores. Overall, SPAT-D scores for the experimental group were lower than scores for the control group, but did not differ statistically. This was likely due to the large variability in experimental group values. Figure 2 shows that the control group participants were minimally variable, and all were at or above the normal mean. Two of three FAS participants produced scores comparable to their matched controls. However, the remaining three FAS participants produced scores sub-

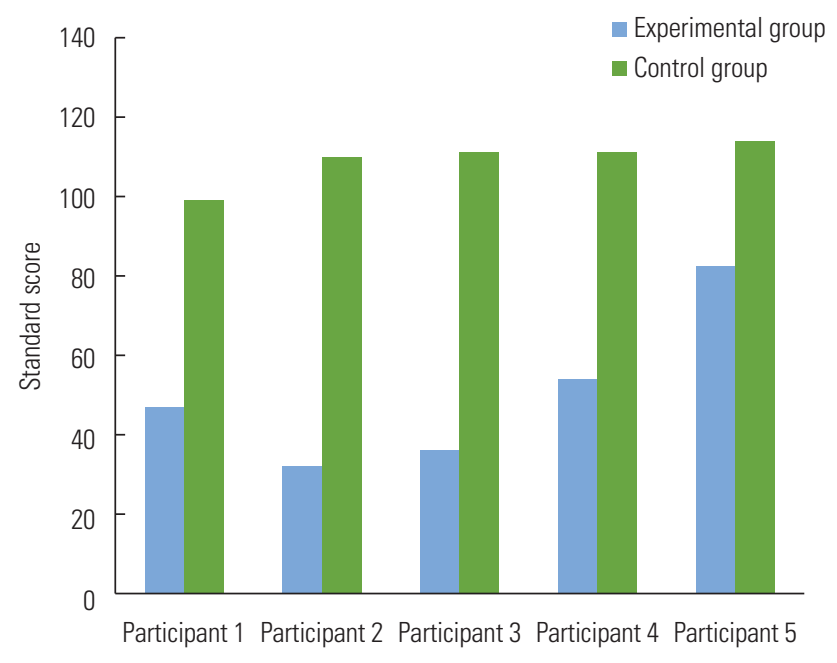

Figure 4. Performance of mental age matched participant pairs on the VMPAC Oromotor Control subtest. All FAS speakers scored lower than their matched controls. VMPAC, verbal motor production assessment for children; FAS, fetal alcohol syndrome.

stantially below those of their matched controls, and substantially below the mean scores for their mental age, indicating articulation errors inappropriate for their mental age.

Figure 3 shows average group scores for each of the $5 \mathrm{VM}$ PAC subtests, expressed as standard scores. Between-group differences were examined for each VMPAC subtest separately. Significant differences were found for three of the five subtests. These were: General Motor Control (ranksum = 17.5, $p=0.0397$ ), Focal Oromotor Control (ranksum $=15, p=$ 0.0079), and Sequencing (ranksum $=19, p=0.00476$ ). Scores for the Connected Speech and Speech Characteristics subtests did not significantly differ.

The most marked group difference was found for the VMPAC Focal Oromotor Control subtest suggesting that nonspeech oral motor control was most markedly abnormal for our sample. Figure 4 shows individual subject values for the VMPAC Focal Oromotor Control subtest. All control participants were at or above the expected mean for their mental age and varied minimally within the group. All experimental participants were substantially below the mean for their mental age, all were substantially below the scores for their mentalage matched controls, and the group as a whole varied substantially more than the control group.

\section{DISCUSSION}

This study sought to examine phonetic and phonemic differences between children with a diagnosis of FAS and typically 
developing children. There are oral-motor function issues as well as cognitive function issues in this particular population. The major hypothesis of this study was that oral-motor deficits in the FAS population will result in unusual patterns of articulation or degrees of misarticulation, compared to those of mental-age equivalent control speakers. This study showed increased misarticulation and inconsistent speech productions in the experimental population relative to mental age matched controls though the primary articulation test was insufficiently sensitive to show some of these misarticulations, and the test scores failed to show a statistically significant between-group difference. Another hypothesis of the study was that children with FAS exhibit atypical verbal motor deficits compared to mental-age equivalent control speakers. Selected results showed that of several speech functions, oral motor function was the most obviously disordered in this FAS sample.

A primary goal of the study was to try to disambiguate disordered motoric and linguistic components of speech production in children with FAS. Though SPAT-D articulation scores did not show a statistically significant group difference, this was likely due to the small sample size and the substantial variability of the FAS group. Three of the five FAS speakers had notably low scores on this test. Phonological patterns evident from the SPAT-D are not reported here, however, they suggest articulatory inconsistencies that do not reflect normal developmental phonological processes. They appear to show notable between-subject variability in both type and degree of articulatory delay, and inconsistent phoneme production within subjects as exemplified by variable articulatory patterns on multiple attempts to produce a given word (i.e., articulatory patterns that did not appear linguistically rule-governed in any consistent way).

Since speech and language have a symbiotic relationship, abnormalities in speech articulation in children with FAS may have a motoric component as well as a linguistic component. It is difficult to tell, based only on an articulation test like the SPAT-D, the extent to which articulation errors arise from cognitive-linguistic immaturity, an unusual phonologic rule system, or motor disorder. The VMPAC sub-tests were administered to assess the experimental participants' verbal-motor abilities. The VMPAC Focal Oromotor Control subtest suggests that the basis for the aforementioned inconsistencies may largely arise from fine oromotor control deficiencies. At the very least, VMPAC scores document consistent oromotor control deficits in this sample and suggest that speech communication problems in this population are due to motor control deficits as well as to language development delays.

Up to this point, deficits within the FAS group have been analyzed. However, FAS speakers could do some things well. It is worth noting that the majority of the experimental group scored within functional limits on the VMPAC Speech Characteristics subtest. Two participants exhibited a deficit in the area of Speech Characteristics. The Speech Characteristics subtest rates six speech characteristics (i.e., pitch, resonance, voice quality, intensity, prosody, and rate) on a binary scoring system. The experimental participants that showed deficits had abnormalities in one of the six characteristics; however, the test construct does not provide for scoring of degree of severity or for temporary abnormalities that will remediate (e.g., seasonal allergies resulting in vocal hoarseness or a cold producing nasal blockage resulting in resonance issues). Parents of both experimental participants with deficits in the area of speech characteristics reported that they were normally “... able to get loud..." or "...not hoarse like this." Therefore, it is assumed that given another opportunity for vocal assessment, all experimental participants would fall within normal functional limits on the Speech Characteristics subtest. Evidence from the present study suggests that the FAS group has deficits in the area of physiological function of the facial muscles used in articulation rather than laryngeal and respiratory function, and that FAS speakers have a relatively good grasp of prosody and suprasegmental features of speech.

\section{Threats to validity}

The current study has validity issues in that the sample size was very small. This limits the amount of information that is generalizable to the FAS population as a whole. Another threat concerns the heterogeneous nature of the disorder, resulting in high variability within the experimental population. The experimental participants showed a high degree of subject-to-subject variability as compared to the control group on both the cognitive assessment and the articulation assessment. Much of the research in the area of social and behavioral science has small samples due to the low prevalence of the studied populations and the heterogeneous nature of the populations studied. One example is Becker et al's [12] study which is the primary published study related to speech articulation. It included only eight children with FAS and suggested oral-motor deficits within the population. Another threat to validity is the failure to control effects of early therapy and intervention in the experimental group. The limited sample size, heterogeneous population, subject-to-subject variability, 
and lack of control for early therapy and intervention threaten validity of the current study; despite this, some consistent trends emerged on standardized measures, and the data still add to the limited FAS knowledge base. However, the small subject sample is not unusual in behavioral studies of the FAS population.

\section{Final thoughts \& future research}

A primary goal of the current research was to disambiguate the motoric from the linguistic component of speech production in children with FAS. The findings strongly suggest a motoric component within the population's deficiencies. This is evidenced by: (1) inconsistencies in phonological findings (i.e., variability in articulation abilities from subject-to-subject, and inconsistent phoneme production within each subject on multiple attempts), (2) deficiencies found in the area of oralmotor control as suggested by the VMPAC Focal Oromotor Function subtest. Further investigation is warranted due to the reduced ability to generalize given the current small sample size, failure to control for previous therapeutic intervention, and heterogeneous nature of the population. Future research might focus on speech motor reaction times, differentiating phonological patterns from inconsistent misarticulations, and efficacy of motor speech therapy to improve articulation in the FAS population. If articulation of children with FAS was studied from a motoric aspect rather than a linguistic aspect, researchers may find that the rule-governed system apparently reflects motor dysfunction rather than anomalies in linguistic-phonologic rules. For example, inconsistent simplifications, such as cluster reductions of a sort that were frequently seen in our FAS sample, might be viewed as primarily motor errors which might remediate through motor development or through motor focused therapy. On the other hand, motorically similar errors, such as gliding of liquids, more likely reflect an immature linguistic rule system. These two categories of articulation errors are addressed differently, but may be dependent upon one another. This research could be applied to other populations with known motor control deficits (e.g., children with cerebral palsy and children with childhood apraxia of speech). The ultimate goal of the chosen direction of research would be to provide clinicians a means of targeting the root problem and not just treating symptoms.

\section{ACKNOWLEDGMENTS}

A special thanks to Dr. Karen Rogers of the West Texas FASD
Diagnostic Team for referring subjects and being available for consultation as needed, Sherry Sancibrian for advice and guidance during the project, Frankie Sims for advising, encouraging, and proofreading, and Judi Keller for encouraging the area of research, and Laura Norman and Bianca Tahuahua for proofreading and formatting assistance. Funding for the project was provided in part through an educational development grant from the American Speech-Language and Hearing Association.

\section{REFERENCES}

1. Lemoine P, Harousseau H, Borteyru JP, Menuet JC. Les enfants de parents alcooliques. Anomalies observées. A propos de 127 cas. Paris Ouest Med. 1968;21:476-482.

2. Ulleland CN. The offspring of alcoholic mothers. Ann N Y Acad Sci. 1972;197:167-169.

3. Jones K, Smith D. Recognition of the fetal alcohol syndrome in early infancy. The Lancet. 1973;302:999-1001.

4. O'Leary C. Fetal alcohol syndrome: diagnosis, epidemiology, and developmental outcomes. J Paediatr Child Health. 2004;40:2-7.

5. Dorrie N, Focker M, Freunscht I, Hebebrand J. Fetal alcohol spectrum disorders. Eur Child Adolesc Psychiatry. 2014;23:863-875.

6. May PA, Gossage JP. Estimating the prevalence of fetal alcohol syndrome. Alcohol Res Health. 2001;25:159.

7. Martin JA, Hamilton BE, Osterman MJK, Driscoll AK, Mathews TJ. Births: final data for 2015 [internet]. United States of America: National Vital Statistics Reports; 2017 [cited 2017 Jan 5]. 70 p. Vol 66, Num 1. Available from: https://www.cdc.gov/nchs/data/nvsr/ nvsr66/nvsr66_01.pdf

8. Coggins TE, Friet T, Morgan T. Analysing narrative productions in older school-age children and adolescents with fetal alcohol syndrome: an experimental tool for clinical applications. Clin Linguist Phon. 1998;12:221-236.

9. Coggins TE, Olswang LB, Olson HC, Timler GR, Abbeduto L. On becoming socially competent communicators: the challenge for children with fetal alcohol exposure. Int Rev Res Ment Retard. 2003;27:121-150.

10. Thorne JC, Coggins TE, Carmichael Olson H, Astley SJ. Exploring the utility of narrative analysis in diagnostic decision making: picture-bound reference, elaboration, and fetal alcohol spectrum disorders. JSLHR. 2007;50:459-474.

11. Popova S, Lange S, Shield K, Mihic A, Chudley AE, Mukherjee RAS, et al. Comorbidity of fetal alcohol spectrum disorder: a systematic review and meta-analysis. The Lancet. 2016;387:978-987.

12. Becker M, Warr-Leeper GA, Leeper HA. Fetal alcohol syndrome: a description of oral motor, articulatory, short-term memory, grammatical, and semantic abilities. J Commun Disord. 1990;23:97124.

13. Church MW, Eldis F, Blakley BW, Bawle EV. Hearing, language, speech, vestibular, and dentofacial disorders in fetal alcohol syn- 
drome. Alcohol Clin Exp Res. 1997;21:227-237.

14. Bolinger C, Dembowski J. Acoustic characteristics of stop consonants in children with fetal alcohol syndrome. Perspectives on
Speech Science and Orofacial Disorders. 2015;25:29-34.

15. Kent RD. Research on speech motor control and its disorders: a review and prospective. J Commun Disord. 2000;33:391-428. 